\title{
GCU
}

Glasgow Caledonian

University

University for the Common Good

\section{PAPR reduction in optical OFDM with grouped LEDs}

Ogunkoya, Funmilayo B.; Popoola, Wasiu O.; Sinanovic, Sinan

Published in:

2016 10th International Symposium on Communication Systems, Networks and Digital Signal Processing (CSNDSP)

DOI:

10.1109/CSNDSP.2016.7573925

Publication date:

2016

Document Version

Author accepted manuscript

Link to publication in ResearchOnline

Citation for published version (Harvard):

Ogunkoya, FB, Popoola, WO \& Sinanovic, S 2016, PAPR reduction in optical OFDM with grouped LEDs. in 2016 10th International Symposium on Communication Systems, Networks and Digital Signal Processing (CSNDSP). IEEE. https://doi.org/10.1109/CSNDSP.2016.7573925

\section{General rights}

Copyright and moral rights for the publications made accessible in the public portal are retained by the authors and/or other copyright owners and it is a condition of accessing publications that users recognise and abide by the legal requirements associated with these rights.

Take down policy

If you believe that this document breaches copyright please view our takedown policy at https://edshare.gcu.ac.uk/id/eprint/5179 for details of how to contact us. 


\title{
PAPR Reduction in Optical OFDM with Grouped LEDs
}

\author{
Funmilayo B. Ogunkoya*,Wasiu O. Popoola ${ }^{\dagger}$ and Sinan Sinanović* \\ ${ }^{*}$ School of Engineering and Built Environment, Glasgow Caledonian University, Glasgow, G4 0BA, UK \\ Email: \{funmilayo.ogunkoya, sinan.sinanovic\}@gcu.ac.uk \\ ${ }^{\dagger}$ Institute for Digital Communications, University of Edinburgh, Edinburgh, EH9 3JL, UK \\ Email:W.Popoola@ed.ac.uk
}

\begin{abstract}
In this paper, we present a study of the combined use of pilot-assisted (PA) electrical PAPR reduction technique and O-OFDM utilising $G$ light emitting diode (LED) groups. In comparison with basic O-OFDM with $G=16$, simulation results show that PA technique is capable of reducing $G$ to 3 in order to achieve the same PAPR of approximately $11 \mathrm{~dB}$ using $P=5$ at complementary cumulative distribution function (CCDF) of $10^{-3}$. The PAPR reduction gain using PA technique is achieved with less hardware complexity, but at the expense of increased computational complexity at the transmitter without sacrificing the bit error rate (BER) performance of the system.
\end{abstract}

Keywords- Optical OFDM; PAPR reduction; Grouped LEDs; Spatial summation; LED transmit chains.

\section{INTRODUCTION}

Wireless communication systems will continue to experience growing demand for high data rates, primarily due to the rapid development of data-intensive applications, services and smart devices. Thus, to meet the demand for high data rates orthogonal frequency division multiplexing (OFDM) has gained much popularity in the field of radio frequency (RF) wireless communication [1]. Motivated by the success of OFDM in RF, OFDM has been adopted in optical wireless communication (OWC) [2]. OWC involves the wireless transmission of data using the optical spectrum. Visible light communication (VLC) which is an indoor OWC, is also gaining popularity due to the advances in light emitting diodes (LEDs) technology. VLC modulates LED intensities for data transmission and uses photodetector as the receiver [2]. Recently, a 3-Gb/s VLC link using optical OFDM (O-OFDM) has been successfully demonstrated with a single gallium nitride LED [3]. In spite of the advantages of O-OFDM, the limited dynamic range of LEDs and other optical front-end devices pose a challenge to its use in OWC due to the inherent high peak-to-average power ratio (PAPR) [4].

The possibility of having individual subcarriers of the OOFDM adding up constructively causes occasional high peaks in the time domain signal. These high peaks imply that the LEDs will have to operate outside its linear region [5]. This mode of operation is undesirable due to the associated distortion present in the transmitted O-OFDM signal [4]. To mitigate such undesirable effects, some form of signal transformation

This work was supported by the School of Engineering and Built Environment of Glasgow Caledonian University under the university sponsored research studentship. technique must be applied to the O-OFDM transmit signal. One approach studied in [6] called predistortion, can be used to maximise the dynamic range of the LEDs. However, the limitation associated with this approach is the turn on and saturation voltage levels of the LEDs. Another approach is to reduce the peak and/or average power of the O-OFDM signal. This approach is categorised as electrical PAPR reduction technique [5], [7]-[9]. Alternative approach reported in [10], [11] is to exploit the LEDs array structure of an LED luminaire to simultaneously transmit subset of the O-OFDM subcarriers. Ordinarily, the PAPR of the O-OFDM signal increases with increase in the number of subcarriers. Thus, mapping subset of the O-OFDM subcarriers to modulate multiple LEDs groups will yield group signals with reduced PAPR. However, increase in the multiple LED groups will imply increasing the number of transmit chains required in the system. In this work, we study the combined use of electrical PAPR reduction technique and multiple grouped LEDs approach to achieve PAPR reduction in O-OFDM while limiting the number of transmit chains required.

O-OFDM utilising multiple LEDs and electrical PAPR reduction technique will provide the opportunity of implementing simplified narrow-band power-efficient LED drivers. Multiple LED grouping technique relies on spatial summation of the intensities of the multiple LED groups during propagation. In practice, LED luminaire usually have closely located multiple LEDs used for illumination due to the limited luminous flux of an individual LED [12]. An indoor OWC channel gain of closely located LEDs has been shown [10], [13] to be static and correlated. Thus, the concept of using the spatial position of LED transmitters as an additional degree of freedom to mitigate some of the existing VLC system limitations is becoming popular. One of such concept has been studied in [13] by utilising spatial summation to eliminate the use of electrical digital-to-analog converter (DAC), thus reducing the design complexity. For spatial summation in $\mathrm{O}-$ OFDM, authors in [11] proposed mapping single O-OFDM subcarrier to exactly one LED. The approach in [10] called spatial O-OFDM (SO-OFDM) uses subset of the O-OFDM subcarriers per LED group.

In this work, we extend the study of the pilot-assisted (PA) technique to reduce PAPR in O-OFDM using multiple LEDs first reported in [14]. While PA O-OFDM system using grouped LEDs is investigated in [14], bit error rate (BER) 
performance of the system is not presented. The signal peak values are reduced in the PA O-OFDM system by rotating the phase of basic data symbols with randomly generated pilot symbol sequence. In this report, we focus on the PAPR reduction gain and BER performance of the PA O-OFDM employing multiple LEDs in the presence of additive white Gaussian noise (AWGN). This study gives a direction for the possible tradeoff between desirable PAPR and complexity of the multiple transmitter O-OFDM system.

The rest of the paper is structured as follows: Section II contains brief description of the basic O-OFDM utilising grouped LEDs. The description of the PA technique with grouped LEDs O-OFDM is contained in Section III. In Section IV, the simulation results with discussions on the performance of the PAPR reduction technique are presented. Finally, conclusions are drawn in Section $\mathrm{V}$.

\section{DESCRIPTION OF BASIC O-OFDM USING GROUPED LEDS}

In VLC, intensity modulation (IM) is employed at the transmitter while direct detection (DD) is used at the receiver. IM requires a real-valued nonnegative baseband signal to modulate the average optical power of the LED. The corresponding information-bearing radiated optical power of the LED depends on factors including the LED efficacy and dynamic range [12]. O-OFDM is a multicarrier modulation technique, implemented by using the computationally efficient inverse fast Fourier transform (IFFT) and fast Fourier transform (FFT). The O-OFDM signal is made up of multiple superimposed orthogonal sinusoidal signals called subcarriers. The superposition of the O-OFDM subcarriers results in occasional high peaks in the time domain signal. These high peaks are characterized using the PAPR metrics. The PAPR refers to the ratio of the maximum instantaneous power to the average power of the O-OFDM signal [15]. O-OFDM signal with $S_{c}$ active subcarriers will have maximum PAPR of $10 \log 2 S_{c}$ (dB). However, the probability of the $S_{c}$ subcarriers adding up constructively to the maximum value at a single point in time is low. Thus, an effective statistical means of charactering PAPR is the complementary cumulative distribution function (CCDF) [15]. The CCDF is the probability that the PAPR is greater than a reference value, $\mathrm{PAPR}_{0}$, which is mathematically expressed as [1]:

$$
\mathrm{CCDF}=\operatorname{Pr}\left\{\mathrm{PAPR}>\mathrm{PAPR}_{0}\right\} .
$$

In basic O-OFDM, an information data stream is mapped to complex symbols, for example, using quadrature amplitude modulation (QAM). Thereafter, the required real-valued OOFDM signal is obtained by imposing Hermitian symmetry on the mapped data symbols prior to loading on IFFT subcarriers. The IFFT operation gives a bipolar real-valued time domain O-OFDM signal. Methods of transforming the resulting realvalued bipolar signal to unipolar signal include: (1) addition of sufficient DC bias, and (2) asymmetrical clipping of the negative part of the signal. The former is termed direct current biased O-OFDM (DCO-OFDM) [16]. The later technique is known as asymmetrically clipped O-OFDM (ACO-OFDM) [17]. After parallel-to-serial conversion and addition of cyclic prefix (CP) to make the signal robust to inter-symbol interference (ISI), the signal is fed into a DAC. Thereafter, the unipolar O-OFDM signal modulates the intensities of the LEDs. Then, all the LEDs simultaneously radiate the same information-bearing optical signal over the VLC channel at a particular time. At the receiving end, a photodetector is used to generate an electrical signal proportional to the intensity of the received optical signal.

On the other hand, a basic O-OFDM employing multiple LED groups approach differs in the manner in which the subcarriers are assigned to the array of LEDs. In this case, the LEDs are grouped while subset of subcarriers are assigned to each group. The least possible PAPR that can be achieved using this approach is when one subcarrier is solely assigned to each LED group. Thus, each LED group transmits a sinusoidal waveform with a PAPR of $3 \mathrm{~dB}$. In a more general form, combination of subcarriers to form subset of the IFFT subcarriers are assigned to modulate intensities of LED groups. Thus, the multiple LEDs transmitter utilises $G$ LED groups to simultaneously transmit subsets of the subcarriers per group. It can be done such that the subcarriers are divided equally among the groups or otherwise. For equal division where $\mathcal{D}$ is the total number of LEDs in the array, the same signal modulates the intensities of $\mathrm{L}_{g}=\mathcal{D} / G$ LEDs per group.

In the basic O-OFDM using grouped LEDs, the data sequence is mapped to QAM symbols and partitioned in the train of $n_{s}$ QAM symbols. Hermitian symmetry is satisfied on the trains of QAM symbols, thereby increasing the train size to $2\left(n_{s}+1\right)$. There are $G$ filtering blocks within the system [10], and Hermitian symmetry is also fulfilled on all the filter vectors $\mathbf{F}_{1}, \mathbf{F}_{2}, \cdots, \mathbf{F}_{g}, \cdots, \mathbf{F}_{G}$, where $g$ is the group number. Hence, each filter vector also has length of $2\left(n_{s}+1\right)$. Then, signal train is simultaneously fed into the distinct $\mathbf{F}_{g}$ filtering blocks. Each $\mathbf{F}_{g}$ narrows down the band of the subcarriers, such that there are $n_{g}=n_{s} / G$ active datacarrying subcarriers per filter output. This is done to ensure the output of each $\mathbf{F}_{g}$ represents distinct subset of the O-OFDM subcarriers. Thereafter, the output of the filter $\mathbf{F}_{g}$ is loaded on the IFFT subcarriers according to the DCO-OFDM or ACOOFDM scheme. The time domain signal from the $g^{t h}$ IFFT modulates the $g^{t h}$ group LEDs. Each LED group has separate driver. It is assumed that the $\mathcal{D}$ LEDs with the same orientation are placed close to one another such that they have nearly identical gains and delays at the receiver. Thus, the system relies on spatial summation of the simultaneously transmitted subcarriers of $G$ LED groups during propagation in space. Hence, it is possible to utilise the photodetector of a basic O-OFDM receiver to detect the optical signal at the receiver.

\section{Pilot-Assisted O-OFDM System With GRouped LEDS}

\section{A. Review of Pilot-assisted PAPR Reduction Technique in $O$ - OFDM}

Figure 1 illustrates the block diagram of the PA OOFDM system. It consists of randomly generated data stream $X_{\mathrm{d}}$ mapped to $M$-QAM symbols, where $M$ is the constellation order. The mapped data symbols are grouped 

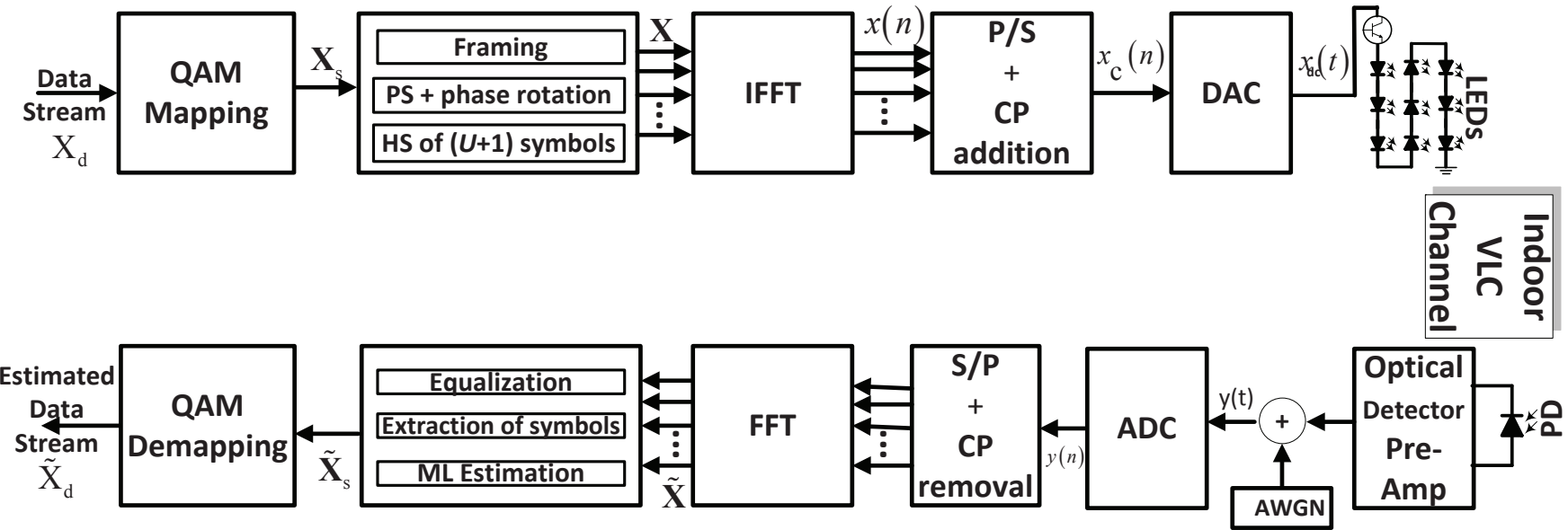

Fig. 1: Block diagram illustrating the pilot-assisted O-OFDM system. PS: pilot symbol; HS: Hermitian symmetry; P/S: Parallelto-serial; S/P:serial-to-parallel; ADC: analog-to-digital converter

into frames of symbols called O-OFDM frame. The frame $X_{\mathrm{s}}^{u}(i), \quad i=1,2, \ldots, n_{\mathrm{s}}, u=1,2, \ldots, U$, with $n_{s}$ being the number of QAM symbols in a symbol train and $U$ is the number of symbol trains in the frame. Thereafter, random sequence of pilot signal $X_{p}(i), \quad i=1,2, \ldots, n_{\mathrm{s}}$ is generated and used in rotating the phase of the $\mathbf{X}_{\mathrm{s}}^{u}$ data symbols. Thus, the pilot symbol increases the PA O-OFDM frame size from $U$ to $U+1$. The data symbol phase rotation is illustrated in (2) as:

$\mathbf{X}_{\text {sp }}=\left(\begin{array}{cccccc}a_{11} / \theta_{11}+\theta_{1 p} & \cdots & a_{1 u} / \theta_{1 u}+\theta_{1 p} & \cdots & a_{1 U} / \theta_{1 U}+\theta_{1 p} & 1 / \theta_{1 p} \\ a_{21} \not \theta_{21}+\theta_{2 p} & \cdots & a_{2 u} \angle \theta_{2 u}+\theta_{2 p} & \cdots & a_{2 U} \angle \theta_{2 U}+\theta_{2 p} & 1 \not \theta_{2 p} \\ \vdots & \vdots & \vdots & \vdots & \vdots & \vdots \\ a_{i 1} \angle \theta_{i 1}+\theta_{i p} & \cdots & a_{i u}\left\langle\theta_{i u}+\theta_{i p}\right. & \cdots & a_{i U} / \theta_{i U}+\theta_{i p} & 1 / \theta_{i p} \\ \vdots & \vdots & \vdots & \vdots & \vdots & \vdots \\ a_{n_{s} 1} \angle \theta_{n_{s} 1}+\theta_{n_{s} p} & \cdots & a_{n_{s} u} / \theta_{n_{s} u}+\theta_{n_{s} p} & \cdots & a_{n_{s} U} \angle \theta_{n_{s} U}+\theta_{n_{s} p} & 1 / \theta_{n_{s} p}\end{array}\right)$

where $a_{i u}$ and $\theta_{i u}$ are the QAM constellation amplitude and phase of the $i^{\text {th }}$ subcarrier and $u^{\text {th }}$ O-OFDM symbol respectively. Also, $\theta_{i \mathrm{p}}$ represents the phase of the $i^{\text {th }}$ pilot symbol which is either 0 or $\pi$ to ease data recovery at the receiver and maintain the original signal constellation. The amplitude of the pilot symbol $\left|X_{\mathrm{p}}(i)\right|=1$ for the purpose of preserving the data signal electrical power [18]. Hermitian symmetry is imposed on the PA O-OFDM frame $\mathbf{X}_{\mathrm{sp}}$. As a consequence, the Hermitian symmetry operation results in an increase of the number of subcarriers from $n_{\mathrm{s}}$ to $N=2\left(1+n_{\mathrm{s}}\right)$ [19]. Oversampling factor $L$ is used to approximate the continuous O-OFDM signal in the discrete time domain [20]. Oversampling in O-OFDM is done with the addition of padding zeros in the frequency domain. Thus, an illustration of the Hermitian symmetry operation on the oversampled PA O-OFDM frame is given by (3):

$\mathbf{X}^{u}=[0, \mathbf{X}_{\mathrm{sp}}^{u}(i), \underbrace{0,0, \ldots, 0}_{N(L-1) / 2}, 0, \underbrace{0,0, \ldots, 0}_{N(L-1) / 2}, \mathbf{X}_{\mathrm{sp}}^{u^{*}}(N L-i)]_{(3)}^{\mathrm{T}}$

where $[\cdot]^{\mathrm{T}}$ denotes transpose of $[\cdot], \mathbf{X}^{*}(\cdot)$ denotes complex conjugate of $\mathbf{X}(\cdot)$, and $N(L-1)$ zeros account for the $L$ times oversampling. Hence, the oversampled time domain PA
O-OFDM signal, $x(n)$, is the IFFT of $\mathbf{X}$ :

$$
\begin{aligned}
x(n) & =\operatorname{IFFT}\{\mathbf{X}\} \\
& =\frac{1}{\sqrt{N L}} \sum_{i=0}^{N L-1} X(i) \exp \left[\frac{j 2 \pi i n}{N L}\right] ; \quad 0 \leq n \leq N L-1
\end{aligned}
$$

where $j=\sqrt{-1}$. Thereafter, the PAPR of $x(n)$ is evaluated.

$P$ iterations of the data symbol phase rotation with randomly generated pilot symbol sequence are done with the PAPR evaluated accordingly. Each iteration leads to a change in the statistical behaviour of the $U$ data symbols. The essence is to select $x(n)$ that avoids coherent addition of the $N$ subcarriers as much as possible. The PAPR reduction of the PA O-OFDM signal is accomplished by selecting the PA OOFDM frame $x(n)$ that gives the least PAPR out of the $P$ iterations.

$\mathrm{CP}$ is appended to $x(n)$ and then fed into a DAC to obtain a continuous time domain signal $x(t)$. Signal $x(t)$ is converted to unipolar signal by adding sufficient bias, before modulating the intensities of the LEDs. The signal applied to the LED driver can thus be expressed as:

$$
x_{\mathrm{dc}}(t)=x(t)+\mathcal{B},
$$

where $\mathcal{B}$ is the bias voltage required to make the signal unipolar. To avoid lower clipping, $\mathcal{B} \geq|\min x(t)|$. Also, the corresponding average optical power, $P_{t}$, of the O-OFDM signal must not exceed the permissible peak power of the LED in its linear region to avoid upper clipping. The channel is modelled as a system with instantaneous input signal $x_{\mathrm{dc}}(t)$ and output signal $y(t)$, the received time domain signal can be written in discrete form as:

$$
y(n)=h(n) \otimes x_{\mathrm{dc}}(n)+w(n),
$$

where $h(n)$ is the discrete channel impulse response and $w(n)$ is the AWGN samples with variance $\sigma_{\mathrm{AWGN}}^{2}$. After the removal of $\mathrm{CP}$, serial to parallel conversion and FFT operation, 
(6) is rewritten in frequency domain as:

$$
\begin{aligned}
& Y^{u}(i)=H(i) X^{u}(i)+W(i) \\
& \quad u=1,2, \ldots, U+1 ; \quad i=0,1, \ldots, N L-1 .
\end{aligned}
$$

By using the zero-forcing (ZF) equalization technique [21], we estimate the noise corrupted PA O-OFDM frame as:

$$
\tilde{X}^{u}(i)=\frac{Y^{u}(i)}{H(i)}, \quad u=1,2, \ldots, U+1 .
$$

The choice of the amplitude and phase of the pilot symbol sequence employed at the transmitter helps in reducing the number of combinations needed to be searched in obtaining the estimated pilot symbol sequence. This approach makes the maximum likelihood (ML) technique suitable for pilot estimation at the receiver [5]. Based on the ML decision rule, the estimate of the received pilot symbol sequence is:

$$
\hat{X}_{p}(i)= \begin{cases}+1 & \text { if } \operatorname{Re}\left\{\tilde{X}_{p}(i)\right\} \geq 0 \\ -1 & \text { otherwise }\end{cases}
$$

where $R e\{\cdot\}$ represents the real part of the pilot symbol and $\tilde{X}_{p}(i)=\tilde{X}^{U+1}(i)$. The recovered active data carrying signal is then denoted as $\tilde{X}_{\mathrm{sp}}^{u}(i) ; i=1,2, \ldots, n_{\mathrm{s}}$. Thus, the transmitted data symbol recovery can be done using (10):

$$
\tilde{X}_{\mathrm{s}}^{u}(i)=\frac{\tilde{X}_{\mathrm{sp}}^{u}(i)}{\hat{X}_{p}(i)} ; \quad u=1,2, \ldots, U .
$$

Lastly, $\tilde{X}_{\mathrm{s}}^{u}(i)$ is demodulated to reconstruct the transmitted data stream $\tilde{X}_{\mathrm{d}}$.

\section{B. Transmitter Architecture of Pilot-assisted O-OFDM System with Grouped LEDs}

Figure 2 represents block diagram illustrating the transmitter of PA O-OFDM system with grouped LEDs. The same procedure of $M$-QAM data symbols phase rotation with $P$ iterations of pilot symbol sequence and Hermitian symmetry as described in Section III-A is followed. The PA O-OFDM

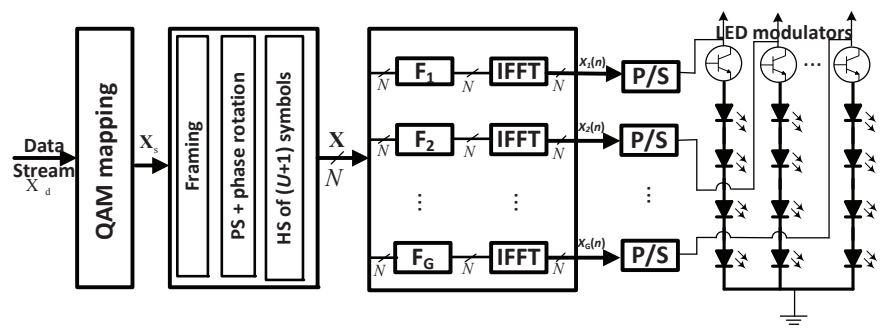

Fig. 2: Block diagram illustrating the transmitter architecture of PA O-OFDM system with grouped LEDs.

frame $X^{u}(i), \quad i=0,1,2, \ldots, N-1, u=1,2, \ldots, U+1$ is fed into the filtering block containing $G$ filters. To ensure the output of the filters represent distinct subset of the PA OOFDM frame, Hermitian symmetry is equally ensured on the frequency domain filter vectors $\mathbf{F}_{g}$ given as [10]:

$$
\mathbf{F}_{g}(i)=\left[0, \hat{\mathbf{F}}_{g}(i), 0, \hat{\mathbf{F}}_{g}^{*}(N-i)\right]^{\mathrm{T}} \quad i=1,2, \cdots, n_{s},
$$

where

$$
\hat{F}_{g}(i)=\left\{\begin{array}{ll}
1 & i=(g-1) n_{g}+1, \cdots, g n_{g} \\
0 & \text { otherwise }
\end{array},\right.
$$

and $n_{g}=n_{s} / G$ represents the number of active data-carrying subcarriers per group. Thus, the $g^{t h}$ filter output $\mathbf{X}_{g}^{u}(i)$ is given by:

$$
X_{g}^{u}(i)= \begin{cases}0, & i=0, \frac{N}{2} \\ F_{g}(i) X_{\mathrm{sp}}(i), & i=1,2, \cdots, \frac{N}{2}-1 \\ F_{g}(i) X_{\mathrm{sp}}^{*}(i), & i=\frac{N}{2}+1, \frac{N}{2}+2, \cdots, N-1 .\end{cases}
$$

where $*(\cdot)$ denotes complex conjugate and DCO-OFDM scheme is in use. Thereafter, the unitary IFFT transform is used to obtain time domain signal $x_{g}(n)$.

With $G=1$, the data symbol phase rotation and minimum PAPR of frame selection process described in Section III-A is analogous to the PA O-OFDM system with grouped LEDs. Thus, the PAPR reduction is achieved by repeating the phase rotation of the $U$ data symbols, $\theta_{i u}$, with $P-1$ more iterations of pilot sequence. This is followed by filtering of the PA OOFDM frame through $G$ filters, and then IFFT operation. Thereafter, the PAPR of the $g^{t h}$ IFFT output is evaluated accordingly. The PA O-OFDM frame that gives the minimum value after $P$ iterations is selected to modulate the $g^{\text {th }}$ LED group. The PAPR of the $g^{t h}$ oversampled time domain signal, $x_{g}(n)$, is evaluated as:

$$
\mathrm{PAPR}=\underset{1 \leq p \leq P}{\arg \min }\left[\frac{\max _{0 \leq n \leq(U+1)(N L-1)}\left|x_{g}(n)\right|^{2}}{E\left[\left|x_{g}(n)^{2}\right|\right]}\right] .
$$

where $E[\cdot]$ denotes the statistical expectation. Addition of appropriate DC bias, $\mathcal{B}_{g}$, to obtain unipolar signal, $x_{\mathrm{dc}_{g}}(n)$, needed to drive the $g^{t h}$ optical modulator results in:

$$
x_{\mathrm{dc}_{g}}(n)=x_{g}(n)+\mathcal{B}_{g} .
$$

Without loss of generality, it is assumed that the LEDs have identical gains and similar delays at the receiver [10], [13], [22]. Hence, the optical group signals radiated by the LEDs sum up in space. It is assumed that the radiated power is less than the saturation optical power of the photodetector which has responsivity of unity. Thus, the received signal, $y(n)$, is proportional to the received power with AWGN noise, that is:

$$
y(n)=L_{g} \sum_{g=1}^{G} x_{\mathrm{dc}_{g}}(n)+w(n) .
$$

The pilot symbol estimation and transmitted data symbol recovery at the receiver remains the same as described in Section III-A. Furthermore, details of the performance of the PA technique in a correlated indoor VLC channel can be found in [9], [18].

\section{RESULTS AND Discussions}

In this section, we present the performance of the PA technique in O-OFDM system with grouped LEDs in comparison with the basic counterpart. We consider $L=4$-times 
oversampled O-OFDM system with $n_{s}=96$ active datacarrying subcarriers and 4-QAM system. To quantify the PAPR reduction gain of the PA technique, the CCDF metric is used. Also, another metric used to evaluate the PA technique BER performance in the presence of AWGN is the optical signalto-noise ratio (SNR) defined as:

$$
\mathrm{SNR}=\frac{P_{\mathrm{opt}}}{\sigma_{\mathrm{AWGN}}}
$$

where $P_{\text {opt }}=L_{g} \sum_{g=1}^{G} P_{\mathrm{opt}_{g}}$ is the total average optical power, $P_{\mathrm{opt}_{g}}$ is the average optical power radiated per group.

Figure 3 compares the PAPR, using CCDF of $10^{-3}$, of basic and PA O-OFDM utilising different $G$ when $n_{s}=96$. In the figure, the number of LED groups $G=1,2,3,6,12,16,24,32,48,96$ and their corresponding number of active data-carrying subcarriers per group are $n_{g}=96,48,32,16,8,6,4,3,2,1$ respectively. It should be noted that $G=1\left(n_{g}=96\right)$ with the maximum PAPR in both basic and PA O-OFDM corresponds to systems where LEDs are not grouped. Results show that PAPR decreases with increasing $G$ for basic and PA O-OFDM employing multiple LEDs. To show the peak reduction capability of the PA technique, the PAPR gains for $G=2,3,6,12,16$ are about $2.3 \mathrm{~dB}, 2.3 \mathrm{~dB}, 2.2 \mathrm{~dB}, 1.9 \mathrm{~dB}$ and $1.4 \mathrm{~dB}$ respectively when compared with the basic O-OFDM with the same $G$. Furthermore, it is noticeable that to reduce the PAPR to approximately $11 \mathrm{~dB}$ using grouped LEDs, the PA system using $P=5$ requires $G=3$ whereas basic O-OFDM needs $G=16$. This illustrates the ability of the PA technique to minimise the required number of LED drivers to achieve desirable PAPR in grouped LEDs O-OFDM system.

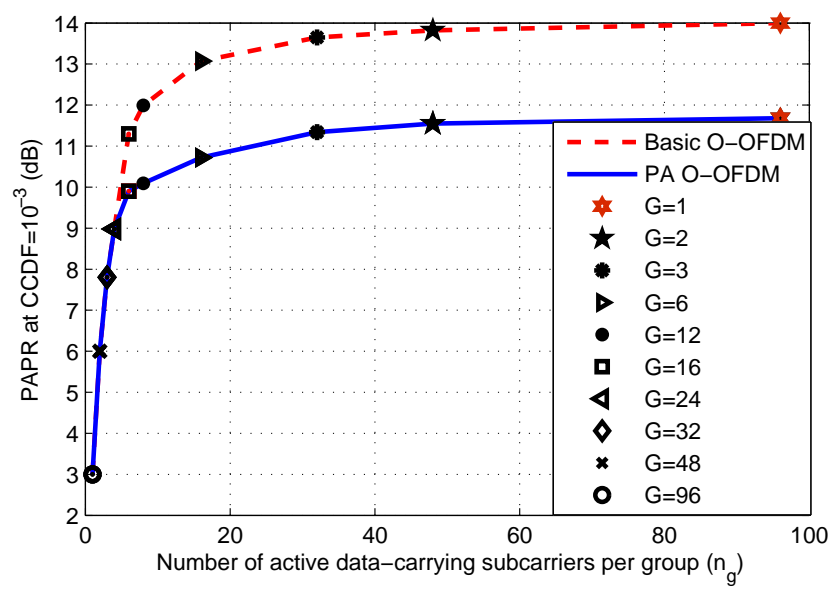

Fig. 3: PAPR required to achieve a CCDF of $10^{-3}$ against the number of active data-carrying subcarriers per LED group, $n_{g}=1,2,3,4,6,8,16,18,48,96$, for basic and PA O-OFDM with $P=5, U=5, \mathcal{D}=96$ and $N=194$

Figure 3 also shows the dependence of the PAPR reduction capability of PA technique on $n_{g}$. The PAPR of basic and PA O-OFDM is the same for $1 \leq n_{g} \leq 4$. This equal PAPR values can be explained by the fact that the pilot signal phase does not change the statistical behaviour of the data symbol to the extent of reducing the PAPR when the number of subcarriers per group is as small as $n_{g} \leq 4$. From the results shown in the figure, PA O-OFDM system must have at least $n_{g}>4$ before it can achieve PAPR reduction gain. As expected, the single subcarrier per LED group $\left(n_{g}=1\right)$, provides the lower bound (minimum possible PAPR of $3 \mathrm{~dB}$ in O-OFDM) for the basic and PA system. It is noteworthy that our simulation upto maximum number of LED groups $G=96$ is for the sake of completeness and to illustrate when the PA technique becomes less effective. In practice, the condition of correlated VLC channel gain may not hold for large $G$, such as $G=96$.

In Fig. 4, we compare the BER performance of basic and PA O-OFDM with grouped LEDs. The BER is plotted as a function of optical SNR for QAM constellation size of $M=4$. Results show that the BER performance of PA O-OFDM system matches that of basic O-OFDM. For low $\mathrm{SNR}$, there is small difference in BER of PA O-OFDM group signals in AWGN when compared with the basic system. This difference can be attributed to the pilot symbol estimation error dominating at low optical SNR while AWGN dominates the system at high SNR. It can be seen that this penalty decreases to zero for BER $<10^{-2}$. This implies that the PA technique does not incur BER penalty on the data symbols at high optical SNR while reducing the PAPR values.

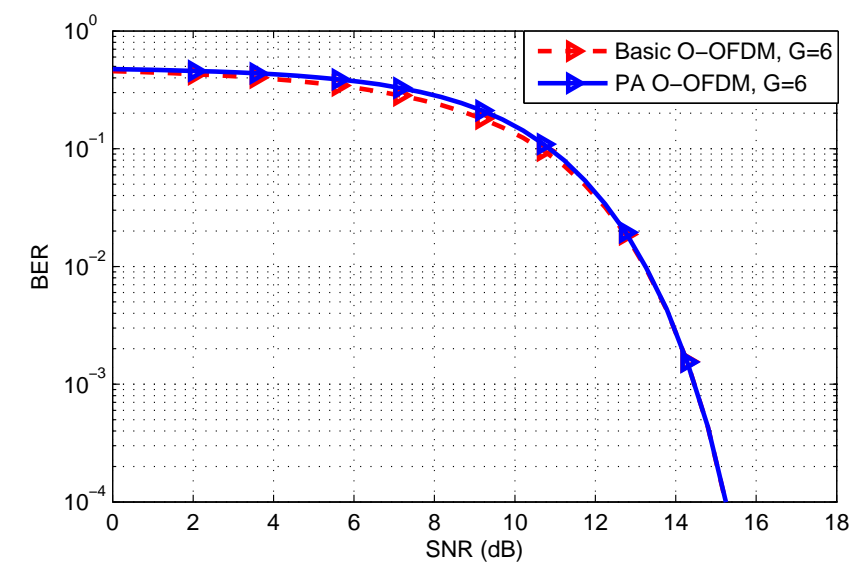

Fig. 4: BER plot of basic and PA O-OFDM with multiple lEDs using $G=6, U=5, P=5, N=194$, 4-QAM

Furthermore, we compare the error performance of the clipped basic and PA O-OFDM signal with and without grouped LEDs in Fig. 5. The signals are subjected to clipping while sufficient DC bias required for the system with and without grouped LEDs are added. We define the normalised upper, $\mathcal{C}_{c_{u}}$, and lower clipping levels, $\mathcal{C}_{c_{l}}$ as: $\mathcal{C}_{c_{u}}=c_{u} \sigma_{x}$ and $\mathcal{C}_{c_{l}}=-c_{l} \sigma_{x}$, where $c_{u}$ and $c_{l}$ are unitless coefficients that determine the severity of clipping experienced at the upper and lower levels of the signal respectively. The clipping generates distortion on all subcarriers and there is addition of clipping noise. The clipping noise dominates a high optical SNR region while AWGN dominates at low SNR. Comparison between the basic and PA system with $G=1$ shows the basic O-OFDM signal to be more susceptible to signal clipping. However, this clipping noise is smaller as $n_{g}$ is reduced since 


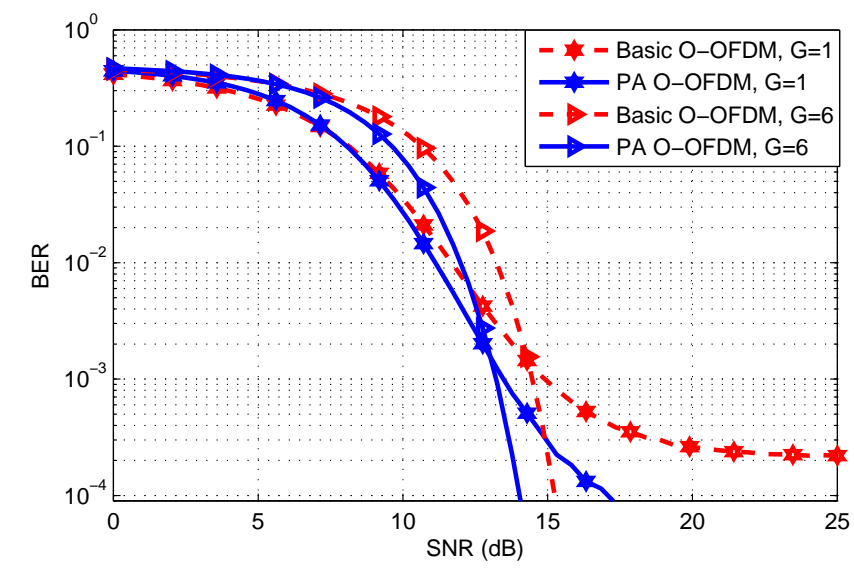

Fig. 5: BER performance of basic and PA O-OFDM system with and without grouped LEDs using $U=5, P=5, G=$ $1,6, \mathcal{D}=96, N=194,4$-QAM. The levels are set to $\sigma_{x}=$ $250 \mathrm{~mA}, c_{u}=c_{l}=1, \mathcal{B}_{g}=250 \mathrm{~mA}$ and $220 \mathrm{~mA}$ for basic and PA system with $G=1$ respectively; while $\mathcal{B}_{g}=40 \mathrm{~mA}$ and $30 \mathrm{~mA}$ for basic and PA system with $G=6$ respectively.

the PAPR values are lower as shown in Fig. 3. Thus, less clipping distortion and consequently, better BER is achieved in the case of basic and PA O-OFDM with $G=6$ at high optical SNR. Combined use of PA technique and grouped LEDs in O-OFDM provides further peak reduction and consequently, reduced DC bias required for the system. Results show that the basic O-OFDM requires more optical SNR to achieve target BER than the PA O-OFDM with grouped LEDs. The PA OOFDM has about $1 \mathrm{~dB}$ optical SNR gain for 4-QAM system at $\mathrm{BER}=10^{-3}$.

\section{CONCLUSION}

In this work, we propose the use of PA PAPR reduction technique in O-OFDM utilising grouped LEDs. In O-OFDM with grouped LEDs, distinct subset of the subcarriers are assigned to modulate intensities of $G$ LED groups. Thus, the grouping approach reduces the number of superimposed subcarriers that can add up to give high peaks in the O-OFDM group signals. However, this peak reduction is obtained at the expense of additional transmit chains which depends on $G$. Hence, PA technique can be implemented in grouped LEDs O-OFDM system to further reduce electrical PAPR, and thus reduce the number of required LED drivers. In comparison, results show that basic O-OFDM group signals with $G=16$ does not have their PAPR values as low as that of PA O-OFDM with $G=3$ using $P=5$ with CCDF of $10^{-3}$. It can thus be inferred that the number of LED drivers can be reduced from 16 to 3 with the PA technique without incurring BER penalty on the O-OFDM signal. When the basic O-OFDM signal utilising multiple LED groups and the PA counterpart are subjected to clipping, results show that the basic O-OFDM signal is more susceptible to BER degradation. It should be noted that PA technique is at the expense of increased IFFT computational complexity. However, recent advances in solid-state technology make it cost effective to implement the iterations in the PA technique.

\section{REFERENCES}

[1] S. William and D. Ivan, OFDM for Optical Communications. Academic Press: Elsevier, USA, 2010.

[2] T. Komine, S. Haruyama, and M. Nakagawa, "Performance evaluation of narrowband OFDM on integrated system of power line communication and visible light wireless communication," in 1st Int. Sym. Wireless Pervasive Computing, 2006, pp. 1-6.

[3] D. Tsonev, C. Hyunchae, S. Rajbhandari, J. McKendry, S. Videv, E. Gu, M. Haji, S. Watson, A. Kelly, G. Faulkner, M. Dawson, H. Haas, and D. O'Brien, "A 3 Gbs Single-LED OFDM-Based Wireless VLC Link Using a Gallium Nitride LED," IEEE Photon. Technol. Lett., vol. 26, no. 7, pp. 637-640, April 2014.

[4] D. Tsonev, S. Sinanović, and H. Hass, "Complete Modeling of Nonlinear Distortion in OFDM-Based Optical Wireless Communication," J. Lightw. Technol., vol. 31, no. 18, pp. 3064-3076, Sept. 2013.

[5] W. O. Popoola, Z. Ghassemlooy, and B. G. Stewart, "Pilot-Assisted PAPR Reduction Technique for Optical OFDM Communication Systems," J. Lightw. Technol., vol. 32, no. 7, pp. 1374-1382, Apr. 1, 2014.

[6] H. Elgala, R. Mesleh, and H. Haas, "A Study of LED Nonlinearity Effects on Optical Wireless Transmission using OFDM," in Proc. $6^{\text {th }}$ IEEE Int. Conf. Wireless and Optical communications Networks (WOCN), Cairo, Egypt, 28-30 April, 2009.

[7] W. Xu, M. Wu, H. Zhang, X. You, and C. Zhao, "ACO-OFDMSpecified Recoverable Upper Clipping With Efficient Detection for Optical Wireless Communications," IEEE Photon. J., vol. 6, no. 5, pp. 1-18, Oct. 2014.

[8] H. Zhang, Y. Yuan, and W. Xu, "PAPR Reduction for DCO-OFDM Visible Light Communications via Semidefinite Relaxation," IEEE Photon. Technol. Lett., vol. 26, no. 17, pp. 1718-1135, Sept. 2014.

[9] F. Ogunkoya, W. Popoola, A. Shahrabi, and S. Sinanovic, "Performance Evaluation of Pilot-assisted PAPR Reduction Technique in Optical OFDM Systems," IEEE Photon. Technol. Lett., vol. 27, no. 10, pp. 10881091, 2015.

[10] M. Mossaad, S. Hranilovic, and L. Lampe, "Visible Light Communications Using OFDM and Multiple LEDs," IEEE Trans. Commun., vol. 63, no. 11, pp. 4304-4313, Nov. 2015.

[11] H. Dong, H. Zhang, k. Lang, B. Yu, and M. Yao, "OFDM Visible Light Communication Transmitter Based on LED Array," Chin. Opt. Lett., vol. 12, no. 5, pp. 052 301-1-4, May 102014.

[12] R. Lenk and C. Lenk, Practical Lighting Design with LEDs. Hoboken, NJ, USA: Wiley, 2011.

[13] T. Fath, C. Heller, and H. Haas, "Optical wireless transmitter employing discrete power level stepping," J. Lightw. Technol., vol. 31, no. 11, pp. 1734-1743, June 2013.

[14] F. B. Ogunkoya, W. O. Popoola, and S. Sinanović, "Pilot-assisted PAPR Reduction Technique for O-OFDM Using Multiple LEDs in VLC Systems," in IEEE International Conference on Communications (ICC'16) Workshop (accepted for presentation), Kuala Lumpur, Malaysia, May 23-27, 2016, pp. 1-6.

[15] Y. Qi, A. A. Abdullah, and S. William, Impact of Nonlinearities on Fiber Optic Communications in Optical and Fiber Communications Reports Vol. 7, S. Kumar, Ed. Springer, 2011.

[16] J. M. Kahn and J. R. Barry, "Wireless Infrared Communications," Proc. IEEE, vol. 85, no. 2, pp. 265-298, Feb. 1997.

[17] J. Armstrong and A. Lowery, "Power Efficient Optical OFDM," Electron. Lett., vol. 42, no. 6, pp. 370-372, Mar. 16, 2006.

[18] F. Ogunkoya, W. Popoola, A. Shahrabi, and S. Sinanovic, "Pilot Symbol Utilization for Reducing Peak-to-average Power Ratio in Optical OFDM," in 9th IEEE/IET Int. Symposium Commun. Systems, Networks and Digital Signal Processing, CSNDSP14, Manchester, UK, July 2014, pp. 899-903.

[19] D. Tsonev, S. Sinanović, and H. Haas, "Novel Unipolar Orthogonal Frequency Division Multiplexing (U-OFDM) for Optical Wireless," in 75th IEEE Veh. Technology Conf. (VTC Spring), Yokohama, 6-9 May 2012, pp. $1-5$

[20] K. Wong, M.-O. Pun, and H. Poor, "The Continuous-Time Peak-toAverage Power Ratio of OFDM Signals Using Complex Modulation Schemes," IEEE Trans. Commun., vol. 56, no. 9, pp. 1390-1393, Sep. 2008.

[21] H. Elgala, R. Mesleh, H. Haas, and B. Pricope, "OFDM Visible Light Wireless Communication Based on White LEDs," in Proc. 64th IEEE Veh. Technol. Conf. (VTC), Dublin, Ireland, Apr. 22-25, 2007.

[22] J. Armstrong, "Optical domain digital-to-analog converter for visible light communications using LED arrays," Photon. Res., vol. 1, no. 2, pp. 92-95, Aug. 2013. 